\title{
GALERKIN METHOD FOR BENDING ANALYSIS OF BEAMS ON NON-HOMOGENEOUS FOUNDATION
}

\author{
Abubakr E.S. Musa \\ Department of Civil and Environmental Engineering, King Fahd University \\ of Petroleum and Minerals \\ Dhahran, Saudi Arabia \\ bakri083@gmail.com
}

Received: 23 February 2017; Accepted: 14 July 2017

\begin{abstract}
In this study, a mathematical formulation for static bending analysis of a beam on a non-homogenous foundation is presented. The proposed method offers an accurate procedure for analysis and design of a beam resting on a varying soil bed. The Winkler foundation model is used and presented using discontinuous functions to account for the sudden change in the soil stiffness coefficient. The solution of the governing differential equation is then obtained using the Galerkin method with the help of approximation functions that satisfy the boundary conditions. A systematic approach for setting the approximation functions for different support and soil conditions is suggested. The accuracy of the proposed method is verified through two numerical examples, and they showed an excellent agreement with the finite element method (FEM) and available literature results.
\end{abstract}

MSC: $65 L 60,74 S 05$

Keywords: Galerkin method, non-homogenous foundation, analysis of beams, beam on elastic foundation

\section{Introduction}

Bending analysis of beams or plates in elastic foundation is used extensively in engineering practice as far as soil-structure interaction is concerned. The backbone of this analysis is modelling the contact pressure between the structural member and the soil bed. Upon the assumed behavior of deformation of the soil under loading, different models are presented to introduce the effect of the soil medium. Some of these models are the one parameter foundation or the Winkler model [1] and two parameters model such as Hetenyi [2] and Pasternak [3] where other springs interacting with the vertical ones are considered.

Analysis of the beam on the elastic foundation model started early on by different researchers [4-8]. Other types of foundation models have also been covered extensively such as a beam resting on a visco-elastic foundation, which has been 
studied by Sonoda et al. [9] and a beam on nonlinear foundation has also been studied [10]. On the other hand, and in addition to the classical beam theory, some researchers went further to study the analysis of Timoshenko's beam on an elastic foundation considering the effect of shear deformation as it was studied in [11-13]. Moreover, the computer coding, as a power of solving many engineering problems, has also been utilized in the analysis of the beam on an elastic foundation as it has been presented by Teodorue [14], who developed a new computer code based on FEM for a beam on an elastic foundation using Matlab software, making it easy to perform different kinds of problems with slight changes in the input data.

In many engineering problems, it is very common to have discontinuity in the loading and the geometrical properties of the beam. Some researchers followed this trend as the study of Yavari et al. [15] for the problem of a beam with loading and geometrical discontinuity. The expression of loading conditions in terms of discontinuous functions was also covered [16] making the treatment of different types of loads easy to handle, and the solution of the governing differential equation was observed using Wolfram Mathematica.

In many construction sites, and without the excessive soil stabilization process, the soil bed is, most likely, found to have different mechanical properties such as bearing capacity and soil subgrade reaction. A comprehensive treatment is, therefore, required to make the soil bed more homogenous. Alternatively, it is very necessary to consider the non-homogeneity conditions of the soil bed in the design stage to account for the associated stresses. This phenomenon of a beam on nonhomogenous foundation was studied and presented [17, 18]. In addition, the design of spread foundation resting on a soil with geological anomaly has also been reported in 2014 [19]. In this latest study, the one dimensional model and the threedimensional model were studied, but in both cases the finite element was used for the analysis process.

In this study, an Euler beam resting on a one-parameter foundation of varying subgrade reaction is presented. The use of discontinuous functions makes it easy to account for a sudden change of subgrade reaction and has a large variety of soil changes within the length of the beam but introduces a new form of difficulty to obtain an analytical solution. The governing differential equation is, therefore, solved using the Galerkin method with a set of approximation functions that satisfy the boundary conditions of the beam. These functions are to be selected using a suggested systematic approach that works for nearly any type of boundary conditions. The results of the proposed method are found to be in an excellent agreement with FEM and the results developed in the literature.

\section{Mathematical formulation}

The governing differential equation of a beam on an elastic foundation is presented in the first part of this section. The Galerkin method and the suggested 
systematic approach for generation of the approximation functions are presented in the second and third parts of this section, respectively.

\subsection{The governing differential equation}

Consider a beam resting on the non-homogenous foundation as shown in Figure 1. The governing differential equation of a beam resting on a foundation with soil stiffness coefficient $k(x)$ and subjected to load $q(x)$ is given by

$$
\frac{d^{4} y}{d x^{4}}+\frac{k(x)}{E I} y(x)=\frac{q(x)}{E I}
$$

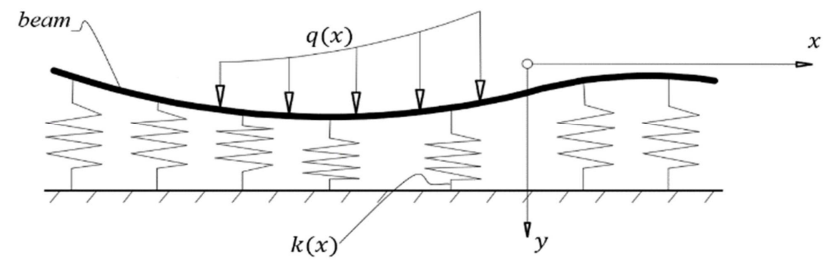

Fig. 1. Typical beam on non-homogenous foundation model

Four boundary conditions are required to solve Eq. (1), and they can be determined according to the physics of the problem. Other beam variables; bending moment $M$, shear force $V$, and slope $\theta$ can be found by the following expressions:

$$
M=\frac{-1}{E I} \frac{\mathrm{d}^{2} \mathrm{y}}{\mathrm{dx}^{2}}, \quad V=\frac{-1}{E I} \frac{d^{3} y}{d x^{3}}, \text { and } \quad \theta=\frac{d y}{d x}
$$

\subsection{Solution formulation}

Let us select the origin of the $x$-coordinate to be at the center of the beam, and let $\hat{y}=C_{j} \emptyset_{j}(x)$ approximate the solution of the of the governing differential equation over the beam domain and $C_{j}$ are constants.

Using the assumed approximated solution $\hat{y}$ in Eq. (1) results in

$$
\frac{d^{4} \hat{y}}{d x^{4}}+\frac{k(x)}{E I} \hat{y}(x)-\frac{q(x)}{E I}=R(x) \neq 0,
$$

where $R(x)$ is the residual. The unknown parameters can be found by setting the weighing average over the computational domain to zero. Leading to 


$$
\int_{-\frac{L}{2}}^{\frac{L}{2}} w_{i}(x) R(x) d \mathrm{x}=0, i, 1,2, \ldots, n
$$

where $w_{i}(x)$ is the weighing function and its selection depends on the selected method of the solution. Using the Galerkin method, $w_{i}(x)=\emptyset_{i}(x)$ where $\emptyset_{i}$ satisfy all the boundary conditions. Eq. (3) can therefore be written as

$$
\int_{-\frac{L}{2}}^{\frac{L}{2}} \emptyset_{i}(x) R(x) d \mathrm{x}=0, i, 1,2, \ldots, n
$$

Use of Eqs. (2) and (4) yields

$$
\int_{-\frac{L}{2}}^{\frac{L}{2}} \emptyset_{i}\left(\frac{d^{4} \hat{y}}{d x^{4}}+\frac{k}{E I} \hat{y}-\frac{q}{E I}\right) d \mathrm{x}=0, i, 1,2, \ldots, n
$$

Substitution of $\hat{y}=C_{j} \emptyset_{j}$ into Eq. (5) and simplifying yields

$$
\int_{-\frac{L}{2}}^{\frac{L}{2}} \emptyset_{i}\left(\frac{d^{4} \emptyset_{j}}{d x^{4}}+\frac{k}{E I} \emptyset_{j}\right) d \mathrm{x} C_{j}=\int_{-\frac{L}{2}}^{\frac{L}{2}} \frac{q}{E I} \emptyset_{i} d \mathrm{x}
$$

which can be written in the following matrix form

$$
[K]\{C\}=\{F\},
$$

where

$$
K_{i j}=\int_{-\frac{L}{2}}^{\frac{L}{2}} \emptyset_{i}\left(\frac{d^{4} \emptyset_{j}}{d x^{4}}+\frac{k}{E I} \emptyset_{j}\right) d \mathrm{x}
$$

and

$$
F_{i}=\int_{-\frac{L}{2}}^{\frac{L}{2}} \frac{q}{E I} \emptyset_{i} d \mathrm{x}
$$

\subsection{Selection of the approximation functions}

The selection procedure of the approximation functions satisfy all the four boundary conditions requiring a clear understanding of the physics of the beam problem under consideration. In this paper, some hints and examples of how to select these functions $\emptyset_{j}$ are given. 
The elastic foundation considered here might take a different form of variation, including a sudden change in the soil subgrade reaction such as presence of geological anomaly in the construction site. The beam can also be partially supported by the soil such as presence of void or a cavity zone under the beam. Modeling of these types of subgrade reactions can be expressed with the help of discontinuous functions such as Heaviside functions. Other forms of variations may include any form of continuous functions. The function that represents the subgrade reaction should be set prior to selection of the approximation functions $\emptyset_{j}$.

Since the approximation functions must satisfy the boundary conditions, let us start by the three beams shown in Figure 2. These three beams have the same zero deflection at their boundaries but differ in the other boundary conditions. This makes it possible to set a systematic approach to find the function $\emptyset_{j}$ for these three cases. Let us introduce a starting function $\bar{y}$ to take the form

$$
\bar{y}=\sum_{k=0}^{m} A_{k}\left(1-4\left(\frac{x}{L}\right)^{2}\right) x^{k}
$$

which satisfies the zero deflection boundary conditions for the three shown cases. The remaining two boundary conditions for each case can be satisfied by forcing the function $\bar{y}$ to satisfy the remaining two boundary conditions. This results in two equations and can be utilized to express two of the constants in terms of the others leading to a function $\bar{y}$ satisfying all the boundary conditions and number of $m-1$ unknown constants $A_{k}$.

Organization of the resultant function $\bar{y}$ and separating it into constants multiplied by functions of $x$ leads to $m-1$ constant multiplied by the same number of functions. Then renaming these functions to be $\emptyset_{j}$ and the constants to be $C_{j}$ for $(j, 1,2, \ldots, n)$ where $n=m-1$. It is noteworthy that $\emptyset_{j}$ satisfying all the boundary conditions and then Eq. (7) can be applied to solve for the unknown $C_{j}$.

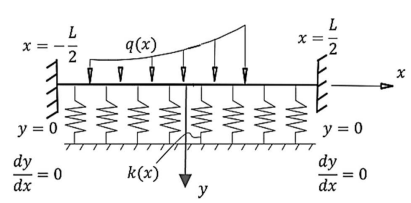

(a) Clamped-clamped beam

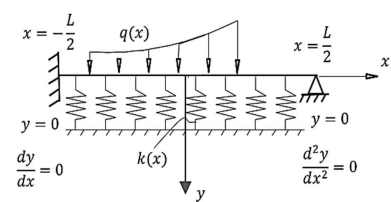

(b) Clamped-hinged beam

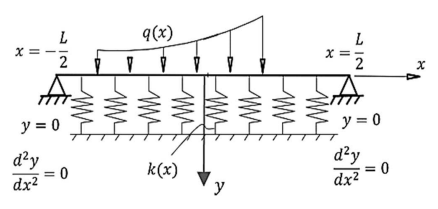

(c) Hinged-hinged beam

Fig. 2. Three beams on non-homogenous foundations and different boundary conditions

One more example is the free-free beam shown in Figure 3. In this problem, due to symmetrical loading and the foundation condition, it is known, as a priori, that the solution will be symmetric, and the beam will have some deflection at the ends. The starting function $\bar{y}$ can, therefore, take the form 


$$
\bar{y}=A_{-1}+\sum_{k=0}^{m} A_{k}\left(1-4\left(\frac{x}{L}\right)^{2}\right) x^{2 k},
$$

where the constant $A_{-1}$ to account for the constant deflection at the ends of the beam. The total number of constants in the above equation is $m+2$. Due to the symmetry property, in this problem, forcing the function $\bar{y}$ to satisfy four boundary conditions results, in fact, in only two equations and, therefore, expressing only two constants in terms of others. Again, organizing the remaining constants and their accompanying functions yields in $m$ number of constants and a similar number of functions. Then, renaming these functions to be $\emptyset_{j}$ and the constants to be $C_{j}$ for $(j, 1,2, \ldots, n)$ and $n=m$.

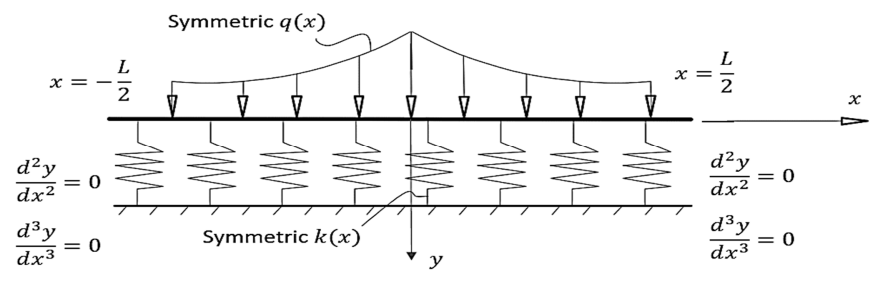

Fig. 3. Free-free beam on non-homogenous symmetrical foundation and subjected to symmetric loading

A similar approach can be used to develop approximation functions $\emptyset_{j}$ for different beam problems. A convergence process is required to decide on the proper number of terms $m$, of the starting function $\bar{y}$, at which the polynomial should be truncated. The convergence procedure has been presented as shown in the next section.

The aforementioned procedure of finding the approximation functions can easily be programed in a small computer code. Then, the obtained approximation functions can be used in Eq. (7) to solve for the unknown variables $C_{j}$. The complete solution $\hat{y}=C_{j} \emptyset_{j}$ can then be found by summing over the dummy index $j$. Other beam variables such as shear force and bending moment can be found using the obtained deflection expression as explained earlier.

\section{Numerical examples}

Application of the developed method has been explained through two numerical examples. The accuracy of the obtained results is verified with those obtained by FEM and the available results in the literature. 


\subsection{Example 1: A foundation of a load bearing wall of finite length constructed on soil of non-homogenous subgrade reaction}

A load bearing wall of total constant uniformly distributed load of $q=100 \mathrm{kN} / \mathrm{m}$ resting on non-homogenous foundation having a subgrade reaction $k$ at the outer quarters and $\alpha k$ at the middle part as shown in Figure 4. The numerical value of the subgrade reaction for the outer quarters per unit length of the foundation is $k=10,000 \mathrm{kN} /\left(\mathrm{m}^{2}\right)$, and the values of $\alpha$ that control the subgrade reaction of the middle part are taken to be $3,2,1$, and 0.75 . The elastic modulus of the material $E=25 \mathrm{GPa}$.
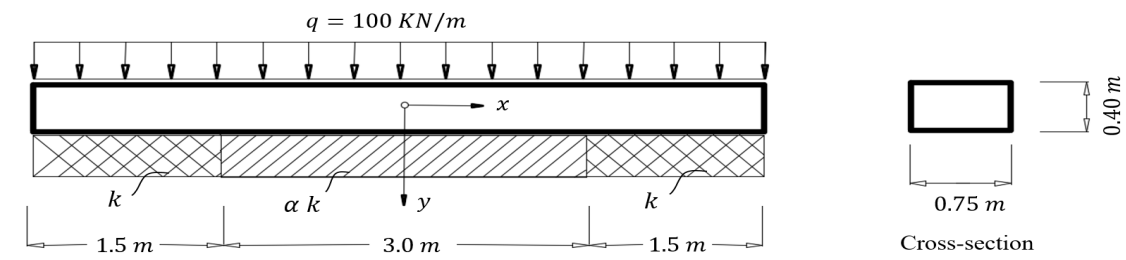

Fig. 4. A foundation of load bearing wall on non-homogenous subgrade reaction

The subgrade reaction modulus, in this problem, can be expressed using the discontinuous function to take the form

$$
k(x)=k+(\alpha-1) k H(x+1.5)-(\alpha-1) k H(x-1.5),
$$

where the discontinuous function $H$ is defined by

$$
H(x-\xi)= \begin{cases}1 & x>\xi \\ 0 & x<\xi\end{cases}
$$

A proper way of modeling this structural member is a free-free beam on nonhomogenous foundation model. As explained in Subsection 2.3, Eq. (9) has been used in this problem as a starting point to find the function $\emptyset_{j}$. A convergence study to decide on the proper number of terms $m$, after which the expression given by Eq. (9) is truncated, has been done for all values of $\alpha$. However, for simplicity, the convergence results have been presented only for the highest and lowest selected values of $\alpha$. From the convergence study, only six terms are found to be enough to truncate the number of terms $m$.

For the purpose of evaluating the accuracy of the proposed method, the results are compared with the FEM prepared model. To reduce the information in the figures, the FEM results are shown for only two values of $\alpha$ ( 3 and 0.75 ). Figures 5 and 6 show an excellent agreement between the proposed Galerkin method and FEM obtained results for the shown two models. The results of the shear force (Fig. 7) show a moderately good agreement with FEM but it is not as excellent as the deflection and bending moment. This less accuracy is due to higher derivatives 
associated with the shear force, which makes the shear force results have the worst agreement with FEM as compared to deflection and bending moment, and also slower convergence rate as shown in Table 1.

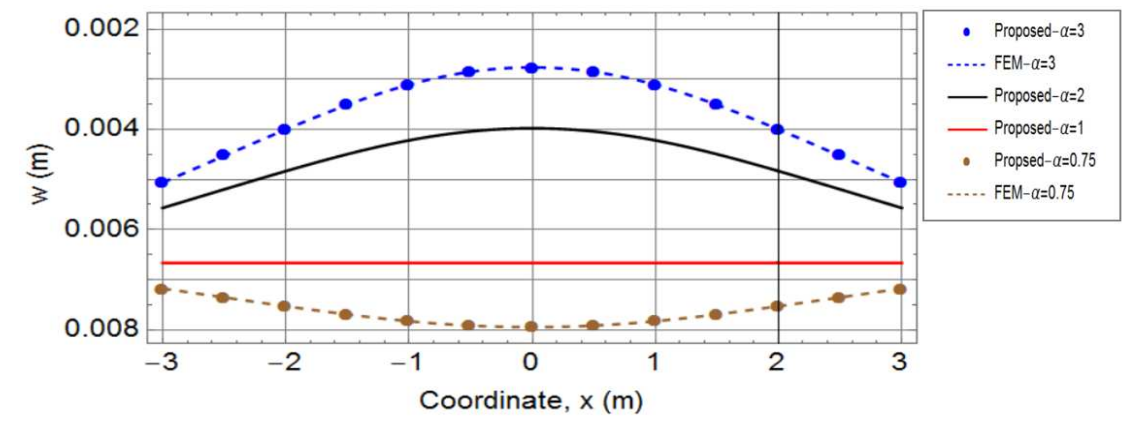

Fig. 5. The deflection of the free-free beam under symmetrically non-homogenous foundation model

Figure 5 confirms that a free-free beam subjected to constant load and resting on a soil of homogenous subgrade reaction $(\alpha=1)$ portrays a constant deflection equal to $q / k$ leading, therefore, to zero bending moment and shear force as shown in Figures 6 and 7. The bending and shear stresses, however, appear with the variation of subgrade reaction along the length of the beam. As a priori construction, the deflection reduces with reduction of subgrade reaction. The results for higher values of $\alpha$ show a low deflection at center of the beam and more deflection as going to the ends of the beam leading to tensile bending stress at the top of the beam. The negative bending moment resulting from higher subgrade reaction at the middle $(\alpha>1)$ as shown in Figure 6 requires reinforcing the top of the beam to account for the resulting tensile stress. Furthermore, the direction of bending moment reverses its direction for $\alpha<1$ leading to a bottom reinforcement requirement with an amount proportional to the reduction of $\alpha$.

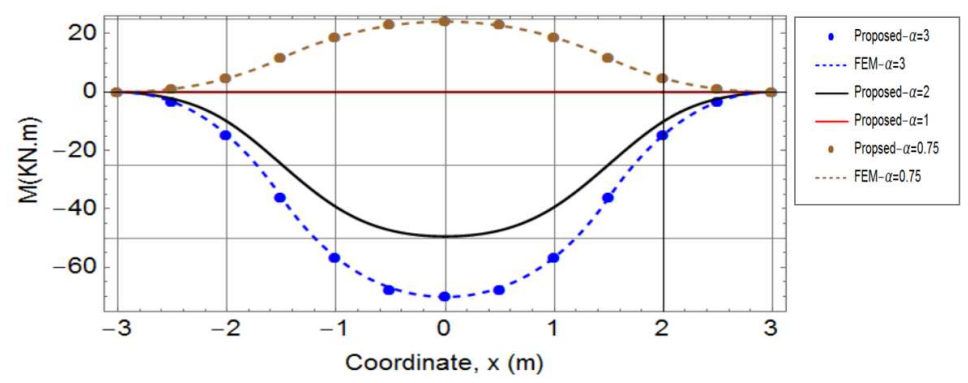

Fig. 6. The bending moment of the free-free beam under symmetrically non-homogenous foundation model 


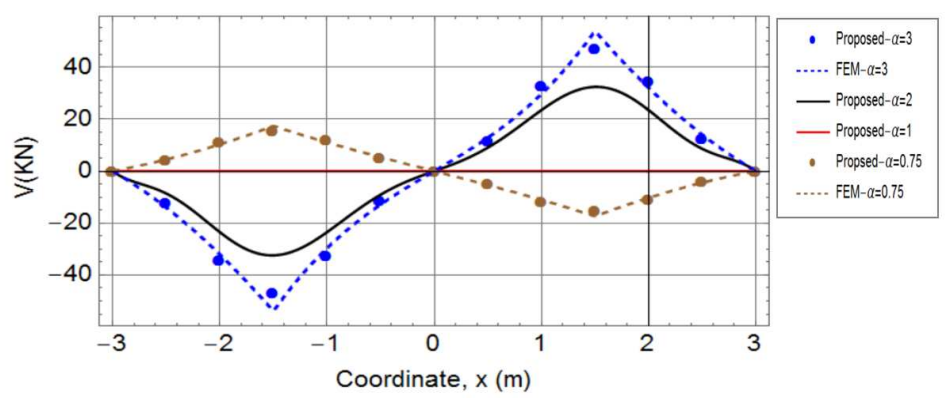

Fig. 7. The shear force of the free-free beam under symmetrically non-homogenous foundation model

Convergence study of Example 1

\begin{tabular}{|c|c|c|c|c|c|c|}
\hline \multirow{2}{*}{$m$} & \multicolumn{3}{|c|}{$\alpha=3$} & \multicolumn{3}{c|}{$\alpha=0.75$} \\
\cline { 2 - 7 } & $\begin{array}{c}y_{x=0} \\
\left(10^{-3}\right)\end{array}$ & $M_{x=0}$ & $V_{x=\frac{L}{4}}$ & $y_{x=0}$ & $M_{x=0}$ & $V_{x=\frac{L}{4}}$ \\
\hline 1 & 3.33 & 0 & 0.00 & 7.62 & 0.00 & 0.00 \\
\hline 2 & 2.78 & -69.47 & 34.73 & 7.94 & 23.20 & -11.60 \\
\hline 3 & 2.77 & -73.10 & 39.12 & 7.94 & 24.81 & -13.51 \\
\hline 4 & 2.77 & -71.40 & 42.50 & 7.94 & 24.42 & -14.25 \\
\hline 5 & 2.77 & -70.28 & 46.73 & 7.94 & 24.11 & -15.42 \\
\hline 6 & $\mathbf{2 . 7 7}$ & $-\mathbf{7 0 . 1 5}$ & $\mathbf{4 6 . 8 2}$ & $\mathbf{7 . 9 4}$ & $\mathbf{2 4 . 0 7}$ & $-\mathbf{1 5 . 4 5}$ \\
\hline
\end{tabular}

\subsection{Example 2: A free-free beam of a partially distributed load resting on non-homogenous foundation}

A free-free beam subjected to a partially distributed load at its middle third and resting on a non-homogenous foundation as shown in Figure 8. The main purpose of presenting this example is to verify the proposed method with one of the available works in the literature as presented by Matsuda and Sakiyama [18], which was developed based on an integral equation and numerical domain integral. The solution is obtained as a function of the applied load $q$, the stiffness of the beam $E I$, and the length of the beam $L$.

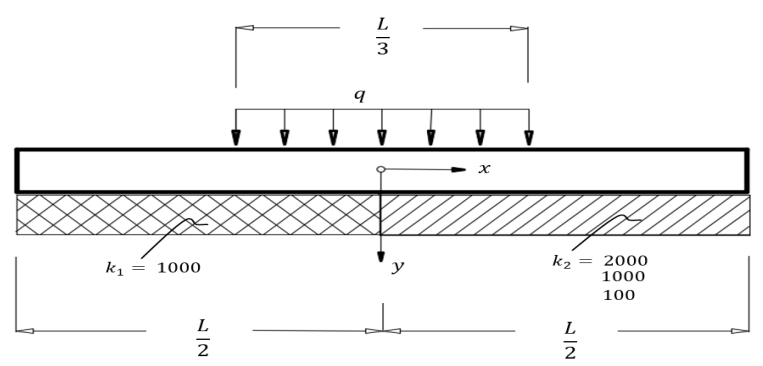

Fig. 8. A free-free beam under non-homogenous foundation model and subjected to a partially distributed load 
The subgrade reaction modulus, in this problem, can be expressed to take the form

$$
k(x)=k_{1}+\left(k_{2}-k_{1}\right) H(x),
$$

where again the discontinuous function $H$ is defined by

$$
H(x)= \begin{cases}1 & x>0 \\ 0 & x<0\end{cases}
$$

A free-free beam problem shown in Figure 8 has asymmetrically arranged subgrade reactions of $k_{1}=1000$ and $k_{2}$ has assigned three values as shown in the figure. This introduces a new form of difficulty in guessing the proper function $\emptyset_{j}$. Therefore, and blindly, a polynomial has been used as a starting function to be

$$
\bar{y}=\sum_{k=0}^{m} A_{k} x^{k} .
$$

Again forcing $\bar{y}$ to satisfy the four boundary conditions and following the same approach to end up with a $K$ matrix of size $(m-3) \times(m-3)$. A convergence study for selecting the proper number of terms $m$ has been studied for the three values of $k_{2}$ and presented in Table 2. It is clear from the table that $m=12$ can be a practical value to truncate the number of terms $m$ for the three values of subgrade reaction.

Figure 9 and 10 show the deflection and bending moment along the beam for the three values of $k_{2}$ in addition to the comparison with the available results in the literature. The comparison shows an excellent agreement between each two counterparts for both the deflection and bending moment.

Table 2

Convergence study of Example 2

\begin{tabular}{|c|c|c|c|c|c|c|}
\hline \multirow{2}{*}{$m$} & \multicolumn{2}{|c|}{$k_{2}=2000$} & \multicolumn{2}{c|}{$k_{2}=1000$} & \multicolumn{2}{c|}{$k_{2}=100$} \\
\cline { 2 - 7 } & $\begin{array}{c}y_{x=0} \\
\left(10^{-3}\right)\end{array}$ & $\begin{array}{c}M_{x=0} \\
\left(10^{-3}\right)\end{array}$ & $\begin{array}{c}y_{x=0} \\
\left(10^{-3}\right)\end{array}$ & $\begin{array}{c}M_{x=0} \\
\left(10^{-3}\right)\end{array}$ & $\begin{array}{c}y_{x=0} \\
\left(10^{-3}\right)\end{array}$ & $\begin{array}{c}M_{x=0} \\
\left(10^{-3}\right)\end{array}$ \\
\hline 1 & 0.242 & 0.000 & 0.333 & 0.000 & 1.217 & 0.000 \\
\hline 5 & 0.242 & 0.000 & 0.333 & 0.000 & 1.217 & 0.000 \\
\hline 6 & 0.440 & 5.980 & 0.617 & 8.209 & 1.334 & 6.448 \\
\hline 7 & 0.447 & 6.121 & 0.617 & 8.209 & 1.380 & 7.016 \\
\hline 8 & 0.468 & 8.450 & 0.640 & 10.709 & 1.398 & 9.201 \\
\hline 9 & 0.469 & 8.462 & 0.640 & 10.709 & 1.400 & 9.231 \\
\hline 10 & 0.469 & 8.948 & 0.640 & 11.222 & 1.402 & 9.655 \\
\hline 11 & 0.471 & 8.943 & 0.640 & 11.222 & 1.402 & 9.660 \\
\hline $\mathbf{1 2}$ & $\mathbf{0 . 4 7 1}$ & $\mathbf{8 . 9 2 9}$ & $\mathbf{0 . 6 4 1}$ & $\mathbf{1 1 . 2 0 9}$ & $\mathbf{1 . 4 0 2}$ & $\mathbf{9 . 6 6 1}$ \\
\hline 13 & 0.471 & 8.929 & 0.641 & 11.209 & 1.402 & 9.661 \\
\hline
\end{tabular}




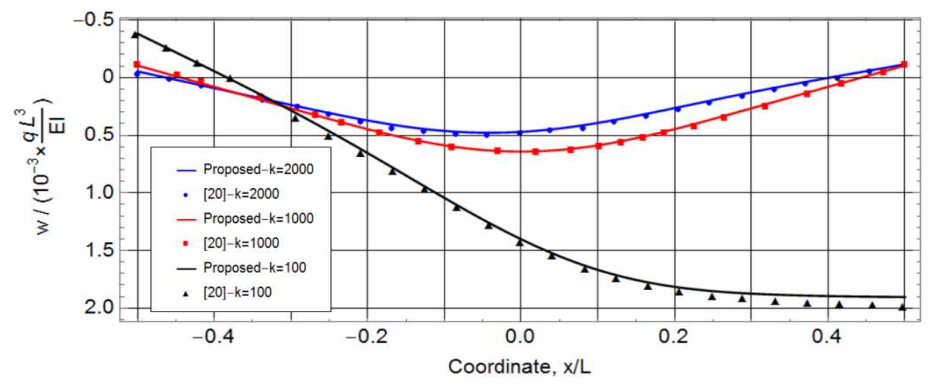

Fig. 9. The deflection a free-free beam under non-homogenous foundation model and subjected to a partially distributed load

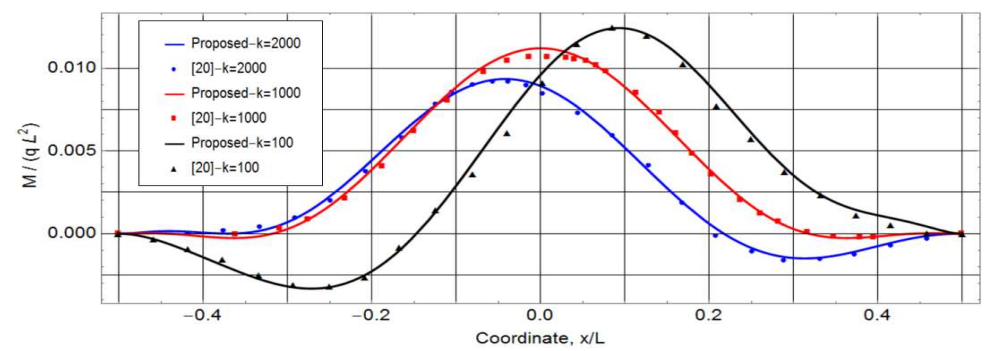

Fig. 10. The bending moment for a free-free beam under non-homogenous foundation model and subjected to a partially distributed load

\section{Conclusions}

A simple solution has been developed for bending analysis of beams resting on a non-homogenous foundation. The solution is based on the Galerkin method and a systematic approach has been described to generate the required set of approximation functions for different beam problems. The accuracy of the proposed method has been verified through two numerical examples of a free-free beam, which are difficult to be solved analytically, and the results proved an excellent agreement with FEM and the available literature results. The proposed method can easily be applied to beams of different supports and soil conditions with no further efforts. The proposed method is of interest for structural engineers who deal with structural design of foundations, especially in construction sites where the soil properties vary considerably.

\section{Acknowledgement}

The author gratefully acknowledges the support provided by King Fahd University of Petroleum \& Minerals for this work. The author is also very grateful to Prof. Husain Jubran Al-Gahtani for his encouragement to finalize this paper. 


\section{References}

[1] Winkler E., Die Lehre von der Elastizität und Festigkeit (The Theory of Elasticity and Stiffness), H. Dominicus, Prague, Czechoslovakia 1867.

[2] Hetenyi M., Beams on Elastic Foundation: Theory with Applications in the Fields of Civil and Mechanical Engineery, University of Michigan Press, Michigan, USA 1946.

[3] Pasternak P.L., On a New Method of Analysis of an Elastic Foundation by Means of Two Foundation Constants, Gosudarstvennoe Izdatelstvo Literaturi po Stroitelstvu i Arkhitekture, Moscow 1954

[4] Krilov A.N., On Analysis of Beams Resting on Elastic Foundation, Sci. Acad. USSR, Leningrad 1931.

[5] Biot M.A., Bending of an infinite beam on an elastic foundation, J. Appl. Mech. 1937, 2, 165-184 .

[6] Miranda C., Nair K., Finite beams on elastic foundation, Journal of the Structural Division ASCE 1966, 92, 131-142.

[7] Ting B.Y., Finite beams on elastic foundation with restraints, Journal of the Structural Division ASCE 1982, 108.3, 611-621.

[8] Teodoru I.B., Analysis of beams on elastic foundation: the finite differences approach, Proceedings of 'Juniorstav 2007', 9-th Technical Conference for Doctoral Study, Brno Univ. Technol. Czech Repulic, January, 2007.

[9] Sonoda K., Kobayashi H., Ishio T., Solutions for beams on linear visco-elastic foundation, Proc. Japan Sot. Civ. Engng. 1976, 247, 1-8.

[10] Beaufait F.W., Hoadley P.W., Analysis of elastic beams on nonlinear foundations, Comput. Struc. 1980, 12.5, 669-676.

[11] Aydogan M., Stiffness-matrix formulation of beams with shear effect on elastic foundation, J. Str. Eng. 1995, 121.9, 1265-1270.

[12] Yin J.H., Closed-form solution for reinforced Timoshenko beam on elastic foundation, Journal of Engineering Mechanics 2000, 126.8, 868-874.

[13] Yin J.H., Comparative modeling study of reinforced beam on elastic foundation, Journal of Geotechnical and Geoenvironmental Engineering 2000, 126.3, 265-271.

[14] Teodoru I.B., EBBEF2p - A Computer Code for Analyzing Beams on Elastic Foundations, Intersections 2009, 6, 28-44.

[15] Yavari A., Sarkani S., Reddy J.N., Generalized solutions of beams with jump discontinuities on elastic foundations, Archive of Applied Mechanics 2001, 71.9, 625-639.

[16] Dinev D., Analytical solution of beam on elastic, Eng. Mech. 2012, 19.6, 381-392.

[17] Al-Gahtani H.J., Finite beams on nonhomogeneous elastic foundations, Bound. Elem. Commun. 1997, 8.3, 168-170.

[18] Matsuda H., Sakiyama T., Analysis of beams on non-homogeneous elastic foundation, Comput. Struct. 1987, 25.6, 941-946.

[19] Imanzadeh S., Denis A., Marache A., Foundation and overall structure designs of continuous spread footings along with soil spatial variability and geological anomaly, Eng. Struct. 2014, 71, 212-221. 